
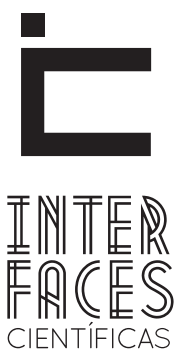

SAÚDE E AMBIENTE

ISSN IMPRESSO 2316-3313

E - ISSN 2316-3798

DOI - 10.17564/2316-3798.2018v6n2p53-64

\title{
CUIDADO DA SAÚDE DOS HOMENS NA ESTRATÉGIA DE SAÚDE DA FAMÍLIA: ESTUDO QUALITATIVO DE RERIUTABA-CE
}

MEN'S HEALTH CARE IN THE “ESTRATÉGIA DE SAÚDE DA FAMÍLIA": QUALITATIVE STUDY OF RERIUTABA - CE

CUIDADO DE LA SALUD DE LOS HOMBRES EN LA ESTRATÉGIA DE SAÚDE DE LA FAMÍLIA: ESTUDIO CUALITATIVO DE RERIUTABA - CE

Mayara Carneiro Alves Pereira ${ }^{1}$

Adriana Alves da Silva ${ }^{3}$
Márbia Araújo Viana²

lização de ações que envolvam os usuários. Contudo, os profissionais reconhecem as possibilidades e potencialidades para que a saúde do homem seja efetivada, faltando ainda iniciativas para desenvolver essa tarefa. Considera-se que esta pesquisa pode fomentar práticas profissionais e ampliar o campo das possibilidades e potencialidades.

\section{PALAVRAS-CHAVE}

gil devido, principalmente, ao pouco domínio de conhecimentos sobre as políticas públicas de saúde, às práticas orientadas por concepções tradicionais com base no senso comum e à falta de iniciativa para a rea-
Atenção Primária à Saúde. Saúde do Homem. Produção do Cuidado. 


\section{ABSTRACT}

The objective of this study is to demonstrate how the professionals with college education and professionals with high school and technical education of Estratégia de Saúde da Família in the Municipality of Reriutaba - CE realize the production of care for men. The study was developed through qualitative research with semi structured interviews and data interpretation performed by content analysis. It was found that the care developed is fragile because, mainly, there is insufficient knowledge about public health policies, the practices are guided by traditional conceptions based on common

\section{RESUMEN}

El objetivo de este estudio es demostrar cómo los profesionales, ya sea los que poseen formación técnica o universitaria, de la Estratégia de Saúde da Família de Reriutaba-CE realizan la producción de cuidados para hombres. El estudio fue desarrollado a través de investigación cualitativa con entrevistas semiestructuradas e interpretación de datos realizada por análisis de contenido. Se verificó que el cuidado desarrollado es frágil porque, principalmente, no hay conocimiento suficiente sobre las políticas de salud pública, las prácticas están orientadas por concepciones tradicionales basadas en el sentido común y en la falta de iniciativa sense and the lack of initiative to make actions that involve users. However, professionals recognize the possibilities and potentialities for men health to be effective, still lacking initiatives for to develop this work. It is considered that this research can foster professional practices and expand the field of possibilities and potentialities.

\section{KEYWORDS}

Primary Health Care. Men's Health. Care Production.

para realizar acciones que involucren a usuarios. Sin embargo, los profesionales reconocen las posibilidades y potencialidades para que la salud de los hombres sea efectiva, aun faltando iniciativas para desarrollar este trabajo. Se considera que esta investigación puede promover prácticas profesionales y expandir el campo de posibilidades y potencialidades.

\section{PALABRAS CLAVE}

Atención Primaria a la Salud. Salud del hombre. Producción del cuidado. 


\section{INTRODUÇ̄̃̃O}

0 presente artigo é fruto de uma pesquisa realizada na Residência Integrada em Saúde (RIS) da Escola de Saúde Pública do Ceará (ESP-CE) que teve como objetivo identificar como os profissionais de uma Estratégia de Saúde da Família (ESF) da zona urbana do município de Reriutaba-CE realizam a produção do cuidado aos usuários do sexo masculino.

Levantamentos nacionais sobre a saúde masculina têm comprovado que os homens são mais vulneráveis às doenças, sobretudo às enfermidades graves e crônicas.Conforme dados apresentados pelo Ministério da Saúde, as causas de morbimortalidade na população masculina,na faixa etária de 25 a 59 anos, incidem em cinco grupos principais, sendo eles, as doenças do aparelho circulatório (20\%), tumores (12\%), doenças do aparelho digestivo (8\%) e do aparelho respiratório (5\%) (BRASIL, 2008).

Morrem mais homens do que mulheres e muitas dessas mortes poderiam ser evitadas se a resistência masculina em procurar serviços de saúde fosse minimizada ou superada. É comum também o menor uso de medicamentos e busca por consultas e exames médicos, quando comparados com as mulheres. No entanto, há um maior número de homens internados em situações graves e com maiores taxas de mortalidade por causas de patologias, como os casos de câncer de próstata, que representam um dos grandes problemas para a saúde pública brasileira (BRASIL, 2008).

Os aspectos socioculturais de virilidade, força e invulnerabilidade dos homens na sociedade brasileira, têm refletido por um lado em uma desvalorização do autocuidado e menor procura aos serviços de saúde e por outro em pouca ênfase das ações dos serviços de saúde para este público.

Realidade semelhante foi constata por Mahalik, Burns e Syzdek (2007) ao realizarem uma pesquisa para verificar aspectos sobre comportamentos de saúde, tais como abuso de substâncias psicoativas, atividades físicas, apoio social, hábitos alimentares e realização de exames anuais. Esses autores (2007) afirmam que o baixo cuidado à saúde do homem, em compara- ção à da mulher, se dá, principalmente, aos padrões normativos associados aos comportamentos de saúde e deste modo concluíram que os modelos tradicionais relacionados ao gênero masculino estão associados aos maiores fatores de risco à saúde masculina, ressaltando assim a importância da promoção da saúde.

Acredita-se que os determinantes sociais de vulnerabilidade do gênero masculino, no que se refere aos hábitos e comportamentos, é também uma condição que necessita de ações efetivas de prevenção e promoção da saúde deste público (BRASIL, 2008).

Frente a essas necessidades, o Ministério da Saúde efetivou a presença dos homens na agenda da Atenção Básica no ano de 2008 por meio da Política Nacional de Atenção Integral à Saúde do Homem (PNAISH), com uma visão holística, que busca ampliar as discussões sobre esta temática e desenvolver medidas que não sejam moralistas e/ou repressivas, entendendo-a como problema de saúde e de interesse do usuário, família e comunidade (BRASIL, 2008).

Em função do reconhecimento da expressividade e necessidade do cuidado com a saúde do homem, o Ministério da Saúde a classifica como uma das dez temáticas a ser priorizada pelas ações da Estratégia de Saúde da Família (SOUZA; RONZANI, 2012). Essa recomendação implica na implementação de cuidado a ser desenvolvido em todos os níveis de complexidade de atenção, incluindo a articulação entre os diversos serviços, dentre eles, os Hospitais, serviços especializados e Atenção Básica, por meio da Saúde da Família.

Diante dessa realidade, algumas inquietações se fizeram sentir no percurso de Residente de Saúde Coletiva da RIS - ESP - CE, como o reconhecimento da importância da Saúde da Família como porta de entrada e ordenadora do cuidado no Sistema Único de Saúde (SUS), a percepção crítica acerca da existência de uma cultura patriarcal mantedora da afirmação da masculinidade, a necessidade de desmistificação da saúde do homem diante das práticas convencionais, resultando na motivação por se investigar como tem sido realizado o cuidado à saúde dos usuários do sexo masculinos na particularidade da cidade de Reriutaba - Ceará. 
Este artigo traz consigo a intenção de contribuir com a identificação e reflexão das concepções profissionais, representando uma proposta bastante desafiadora, pois revela e discute a necessidade de visitar uma combinação de temáticas muitas vezes consideradas periféricas. E parte do princípio de que a produção do cuidado transcende a mera realização de procedimentos e implica o reconhecimento do usuário como sujeito de direitos.

\section{PERCURSO METODOLÓGICO}

\subsection{NATUREZA, LOCAL E PARTICIPANTES DO ESTUDO}

O estudo se realizou por ocasião da Residência Integrada em Saúde (RIS) com ênfase em Saúde Coletiva da Escola de Saúde Pública do Ceará, desenvolvida no período de maio de 2015 a fevereiro de 2016. A investigação norteou-se pela abordagem qualitativa com tipo de estudo descritivo.

A pesquisa aconteceu no município de Reriutaba, cidade do interior do Estado do Ceará e um dos lócus das atividades da RIS. 0 município de pequeno porte se localiza no Noroeste do Estado, possui $383,3 \mathrm{Km}^{2} \mathrm{e}$ conta com uma população de 19.460 habitantes, conforme censo populacional do Instituto Brasileiro de Geografia e Estatística (IBGE, 2010).

O sistema de saúde de Reriutaba-CE possui oito Unidades Básicas de Saúde da Família, sendo elas, três na zona urbana (Sede I, II e III) e cinco na zona rural (Mufumbal, Oiticica, Campo Lindo, Riacho das Flores e Amanaiara). Possui, também, como serviços de saúde um hospital local, um centro de reabilitação de fisioterapia e um Núcleo de Apoio à Saúde da Família (NASF) que atua em articulação com duas academias da saúde.

A pesquisa foi realizada em uma equipe da Estratégia de Saúde da Família da zona urbana do município, tendo como participantes os profissionais: médico (01), enfermeira (01), técnica em enfermagem (01) e uma Agente Comunitária de Saúde (ACS), esta escolhida por sorteio.

\subsection{PROCEDIMENTOS DE PRODUÇ̃̃O, REGISTRO DOS DADOS E ANÁLISE DE DADOS}

Foram procedimentos de produção dos dados, entrevistas semiestruturadas com os profissionais, previamente agendadas e realizadas pelo pesquisador residente em saúde coletiva com autorização dos participantes para gravação.

Os dados advindos das entrevistas foram interpretados pela análise de conteúdo temática, proposta por Minayo (2006), onde se buscou a descoberta de núcleos de sentido da comunicação de cada depoimento e posteriormente foram observados os pontos comuns, divergentes e complementares nas falas dos profissionais, tendo como referência os objetivos da pesquisa.

A discussão das análises foi organizada em quatro categorias que serão apresentadas na próxima seção deste artigo, sendo elas: Concepção de gênero e de saúde do homem; O cuidado na ESF: um olhar para os usuários masculinos; Dificuldades e desafios; Possibilidades e potencialidades.

\subsection{ASPECTOS ÉTICOS E LEGAIS}

A pesquisa foi conduzida dentro de padrões éticos, obedecendo ao disposto na Resolução 466/12 do Conselho Nacional de Saúde (CNS), da Comissão Nacional de Ética em Pesquisa (CONEP) e do Ministério da Saúde (MS) no que diz respeito à pesquisa empírica com seres humanos (BRASIL, 2012). E foi previamente aprovada pelo Comitê de Ética em Pesquisa da Escola de Saúde Pública do Ceará (ESP/ CE), com o número de parecer 1.386 .583 de 11 de janeiro de 2016.

\section{RESULTADOS E DISCUSSÃO}

\subsection{CONCEPÇÃO DE GÊNERO E DE SAÚDE DO HOMEM}

A concepção de gênero oriunda da análise dos depoimentos dos profissionais pesquisados é limitada, ligada à característica biológica e papel cultural na 
sociedade. Percebe-se que, para os profissionais, existe um lugar simbólico de homem como chefe-provedor do lar, geralmente associado ao trabalho. São depoimentos que ilustram essa constatação:

Homem é sexo masculino que se considera homem (Profissional 01);

[...] pessoas que nascem no sexo masculino e se identificam como tal (Profissional 04);

Homens são aqueles que "trabalham o dia todinho" (Profissional 03).

Pensar em gênero é discutir um longo processo histórico e cultural, permeado por lutas e mudanças, passando a ser visto como uma categoria atravessada por questionamentos sobre classe social, raça, etnia, cultura e gerações, sendo um construto que condiciona a percepção do mundo e uma forma de reprodução do que pertence ao masculino e ao feminino. Logo, a tarefa de definir gênero é complexa, pois apresenta vários significados e sentidos sociais e históricos (MACHIN et al., 2011).

A concepção de gênero dos profissionais se assemelha ao encontrado em pesquisa de Camargo et al. (2011) quando estes também colocam que os conceitos naturalizados de homens e mulheres são percebidos de uma forma tradicional, em que o primeiro, sinônimo de produção social, deve ser rapidamente afastado de qualquer possibilidade de doença que prejudique seu vigor no trabalho. Já a mulher, entendida como ser que permanece nos cuidados da casa, percebe seu corpo ser reduzido ao papel reprodutor e cuidador, ou seja, envolto de diversos dispositivos disciplinares.

Deste modo, os cuidados com os homens são sempre vistos como urgência, já que seu papel está associado ao trabalho, o que não implica, muitas vezes, em investigação e tratamento, porém são paliativos para retorno rápido às atividades cotidianas.

0 fator gênero deve ser percebido como um aspecto de grande importância no padrão dos riscos de saúde, diante desse aspecto e da forma como homens e mulheres percebem e usam seus corpos, a maioria das doenças passa a ser entendida como parte de uma combinação entre causas biológicas e socioculturais, uma vez que os processos sociais referentes ao gênero produzem diferenças no padrão de morbimortalidade e proteção à saúde masculina e feminina.

É fundamental que o serviço de saúde esteja atento a essas concepções, visando não apenas desmistificá-las, mas também realizar planejamento das ações com respeito a essas, de modo a envolver os diversos públicos na promoção e prevenção aos riscos e agravos em saúde (SCHRAIBER, 2012).

Relativo à compreensão de cuidados em saúde relacionados aos homens, percebe-se uma conformação desta visão ao papel desempenhado por eles na sociedade. Embora os entrevistados demonstrem que o cuidado é importante, apenas ressaltam que os homens são resistentes, sem qualquer maior explicação ou argumentação, situação que leva a inferir que esses profissionais ainda não conseguem visualizar a saúde deste gênero sob um enfoque mais integral.

Uma das grandes questões de gênero é a dos homens se considerarem invulneráveis e superiores diante dos problemas de saúde, retardando ao máximo a busca por assistência, alegando não terem tempo para cuidarem da saúde, reclamando da constante ausência dos membros da equipe, das longas filas de espera, da falta de medicamentos e a baixa qualificação e atendimento por parte dos profissionais. Com efeito, a visão por esse ângulo ocasiona os adiamentos de consultas e exames, fatores esses que contribuem para uma maior probabilidade de doenças que poderiam ser evitadas (SCHRAIBER et al., 2010).

Cabe destacar que a limitação na concepção de saúde do gênero masculino dos profissionais é fortalecida pela realização de campanhas e por seu caráter pontual e circunstancial, que acabam por fragmentar o trabalho e a percepção dos profissionais no cotidiano. Exemplo dessa situação são as campanhas de Novembro Azul.

A reprodução de uma marca cultural posiciona de maneira binária, o masculino e o feminino, reforçando a existência do modelo tradicional de masculinidade com a ideia que o homem só procura o serviço com problemas graves ou quando impossibilitados de 
trabalhar. Desse modo, ainda que exista uma extensa reflexão da perspectiva de gênero em relação à promoção e à prevenção da saúde na sociedade, ainda são deficientes as referências do modo como os profissionais percebem os usuários do sexo masculino.

\subsection{CUIDADO NA ESF: UM OLHAR PARA OS USUÁRIOS DO SUS DO SEXO MASCULINOS}

Os profissionais pesquisados reconhecem as potencialidades no que se refere aos recursos humanos, infraestrutura e organização dos serviços para o atendimento do usuário do sexo masculino, no entanto, estas propostas não são efetivadas, uma vez que as atividades e programas têm como foco a saúde da mulher, esquecendo, muitas vezes, as especificidades dos homens.

Nas falas dos profissionais essas especificidades estão relacionadas à urologia, com valorização da próstata, revelando o desconhecimento de uma visão ampla de saúde do homem e a presença de conceitos estereotipados.

Nas questões que envolvem o uso de substâncias psicoativas, a percepção dos profissionais é semelhante à de gênero, ou seja, se faz pelo senso comum e sem uma reflexão mais elaborada, fortemente relacionada aos aspectos morais e biológicos, sendo inclusive observado em depoimento dos entrevistados o uso de termos como "bebinhos" e "drogado".

Em suas práticas profissionais, embasadas, muitas vezes, no senso comum e na ausência de conhecimento aprofundado, a equipe desenvolve "medo" diante da relação com os usuários de substâncias psicoativas, o que resulta em um distanciamento na relação entre profissional e usuário do SUS, como pode ser visto nas seguintes falas:

[...] muitas vezes a gente desenvolve um trabalho até de risco, a gente vai na casa de um que está utilizando, a gente está correndo um risco (Profissional 02);

No caso de drogas eles chegam querendo agredir a gente (...) eu acho perigoso, eu ficava com medo, só entrava na casa dele com o motorista [...] fico com medo, porque ninguém sabe qual a intenção deles para com a gente (Profissional 03).

Há também profissionais que reconhecem a importância de um cuidado bem desenvolvido e articulado, como destaca o Profissional 04, que mesmo reconhecendo as dificuldades, apresenta uma forma de atender esse público:

Não é dada a importância ideal de trabalhar a saúde do homem e a esses usuários de álcool e drogas. 0 maior desafio é ter uma base, é saber como dar continuidade com esse paciente. Por aqui a gente não tem essa referência, não tem CAPS AD, nem CAPS comum, então, por não ter essa referência, fica solto, fica um acompanhamento incompleto.

Destaca-se que a proposta de ampliar a oferta de atendimento e incentivar práticas de detecção precoce, prevenir os danos causados pelo uso abusivo e compartilhar os cuidados e responsabilidade pelos usuários em outros serviços são fundamentais na assistência pela ESF. No entanto, nota-se tanto na literatura, quanto no processo dessa pesquisa, a pouca ênfase dada pela ESF para intervenção nos casos de uso prejudicial de álcool e outras drogas, principalmente aos indivíduos do sexo masculino. Essas ações, quando existentes, são mais direcionadas às mulheres gestantes (CRP, 2011).

\subsection{DIFICULDADES E DESAFIOS}

No cotidiano do serviço da saúde pública de Reriutaba-CE foi perceptível as dificuldades e desafios dos profissionais para a produção do cuidado aos usuários do sexo masculino. Uma das dificuldades é a falta de conhecimento dos profissionais, quanto às políticas de saúde e mais especificamente a Política Nacional de Atenção Integral à Saúde do Homem (PNAISH), que pode acarretar em uma posição pouco crítica das dificuldades que envolvem a atuação profissional.

0 segundo entrave é o fato de este público não procurar o serviço,como cita o Profissional 03 "os homens não procuram, vão deixando passar”, e também 
não é procurado pela ESF, com exceção das campanhas de saúde pontuais.

Schraiber (2012), em um estudo acerca da perspectiva das práticas profissionais, observa o conflito dos profissionais em estarem cada vez mais constituindo uma visão do cotidiano dos serviços que opera por meio de uma separação de práticas entre os saberes profissionais, reduzindo-se às questões biomédicas e automatizadas, fugindo das diversas questões socioculturais, entre elas as de gênero. Agindo assim, o profissional se limita a seus juízos morais associados às tradições, fugindo ou até mesmo desconhecendo a real proposta das políticas públicas.

Como desdobramento das concepções de gênero masculino (dos profissionais), destaca-se as falhas de suas atuações, quando se referem ao cuidado aos usuários em uso abusivo e dependência de álcool e outras drogas. 0 depoimento do Profissional 01 traz 0 olhar para esse aspecto, pois "é muito difícil que esse paciente venha à unidade de saúde e também é muito difícil fazer consulta com dependente de drogas".

Essa declaração denota a dificuldade de trabatho e está associada à cultura biomédica reducionista, podendo resultar em práticas de saúde que agravam os processos de exclusão social. Schraiber (2012) enfatiza que se não houver mudanças nesse aspecto, a "visibilidade" que é destinada aos gêneros pode resultar em um esvaziamento ético e político deste conceito.

Outra dificuldade apontada é o trabalho em equipe associado a sobrecarga de trabalho, como retrata o profissional 01, "a maior dificuldade do meu trabalho, da Atenção Básica, é trabalhar em equipe, porque deve haver atendimento, acolhimento [...] e quando a coisa não funciona bem eu brigo muito", outros depoimentos também ratificam a questão:

O tempo é muito pouco, a equipe está sobrecarregada [...] toda hora são muitas demandas (Profissional 01);

Todo tempo tem as necessidades que ficam se sobrepondo, a gente tem as emergências, vem dengue, depois vem as campanhas que tem que cumprir [...] e quando vem o homem é só aquele pedacinho do no- vembro azul e morre ali, é uma coisa que não é dado continuidade. 0 problema é que a gente está meio que apagando incêndio, não está fazendo a promoção que é pra ser, a gente detecta patologias, agravos e fica só remediando (Profissional 04).

A saúde deve então ser compreendida como uma tarefa muito complexa diante da diversidade de conceitos e práticas realizadas. Para além da ausência de doenças, envolvendo questões territoriais, epidemiológicas, sociais, culturais e psicológicas. Nessa direção, deve existir ampliação do conceito de saúde, das suas ações, buscando impulsionar o desenvolvimento de tecnologias relacionais e do compromisso ético e político. Existindo subsídios para incluir, no mínimo, educação em saúde, nutrição, saneamento básico, planejamento familiar, imunizações, prevenção e controle de doenças endêmicas, medicamentos essenciais, além de uma articulação em rede (SCLIAR, 2007).

\subsection{POSSIBILIDADES E POTENCIALIDADES}

Assim como foi dito acerca das dificuldades e desafios, as possibilidades e potencialidades também foram apresentadas indiretamente pelos depoentes, sendo necessário um espaço singular para esta reflexão, principalmente, para se pensar a saúde do homem não somente como falhas, mas construir (conjuntamente) as possibilidades, cabendo também ao pesquisador esse importante papel.

A primeira potencialidade elencada pelos profissionais entrevistados se refere aos aspectos institucionais, estrutura física e recursos humanos. Para estes profissionais a Unidade de Saúde dispõe de recursos humanos da área de saúde (médico, dentista, enfermeiro, técnicos de enfermagem e ACS), que podem organizar serviços e ações para atendimento ao usuário masculino.

A segunda possibilidade é a articulação entre serviços, não apenas na rede de atenção à saúde, com referência para a atenção especializada, mas também com a comunidade, como Alcoólicos Anônimos (AA), como pode ser percebido na seguinte fala: 
[...] aqui em Reriutaba tem o AA, mas eles têm resistência até de procurar o AA porque eles acham que podem parar na hora que quiser. Então, eles não procuram nenhuma ajuda tipo o AA e nem a Atenção Básica (Profissional 02);

Como terceira possibilidade está a intersetorialidade e a interdisciplinaridade para o desenvolvimento do cuidado à saúde onde o Profissional 04 cita a articulação com equipamentos de outras secretarias municipais e com as famílias e o Profissional 01 compreende que:

Essas doenças são mais interdisciplinares, você tem problema de alcoolismo, tem dependência de drogas, posso visitar e conversar com a família. Mas daí para frente tem que entrar a psicóloga, tem que entrar psiquiatra, tem que entrar as enfermeiras, tem que entrar os agentes comunitários de saúde.

Esse profissional destaca a importância de se trabalhar com os diversos saberes no campo da saúde mental, nesta mesma direção, o Profissional 02 relata que fez capacitação onde a interdisciplinaridade foi destacada, situação que demonstra que mesmo existindo um conhecimento frágil, a informação e a necessidade destas práxis é sentida pelos profissionais:

Depois que comecei a trabalhar fiz uma capacitação aqui em Reriutaba que se chamava caminhos do cuidado que era toda voltada para os usuários de drogas, de como é que a gente deve abordar esse paciente, de como a gente deve conversar com as famílias. Foi muito bom, muito proveitoso.

As práticas interdisciplinares, com efeito, são importantes e se apresentam relacionadas principalmente às tecnologias leves/relacionais, que consistem, segundo Mehry (2004) em um trabalho mais vivo e por ocorrer de forma mais aberta e em constante processo, sendo também percebida e referida pelos profissionais como o vínculo, 0 atendimento humanizado, o acolhimento, dentre outras práxis. Como pode ser visto nas seguintes falas:
Deve haver um bom atendimento, acolhimento [...] possibilidade de encaminhamentos para se trabalhar melhor com o problema e do trabalho em equipe. Mas a equipe tem que estar bem preparada, um bom atendimento com o médico, a enfermeira e o agente comunitário de saúde, saber conversar com a pessoa, fazer um grupo de alcoólatra, de dependentes de drogas, um trabalho geral da equipe de saúde [...] tem que ser com uma equipe multidisciplinar, com psicólogo, psiquiatra, Atenção Primária. Um trabalho em equipe, essa atenção tinha que ser feita também com o NASF, com atendimento compartilhado, tem que haver uma equipe, Atenção Básica com o NASF, principalmente com psicólogo (Profissional 01);

[...] desenvolvimento e o atendimento do psicólogo, do psiquiatra, da equipe multiprofissional, eu acho que no acolhimento, na escuta inicial e nas orientações (Profissional 04).

Nota-se que os profissionais acreditam na importância das práticas não tradicionais no cuidado desses homens como acolhimento, atendimento humanizado e compartilhado, visita domiciliar, necessidade de grupos e a participação do Núcleo de Apoio à Saúde da Família.

O NASF objetiva ampliar a abrangência e o escopo das ações da atenção básica, bem como sua resolubilidade, é constituído por profissionais de diferentes áreas de conhecimento, que devem atuar de maneira integrada, apoiando os profissionais da ESF e Academia da Saúde, compartilhando as práticas e saberes em saúde nos territórios, atuando diretamente no apoio matricial às equipes. Fazem parte da atenção básica, mas não se constituem como serviços com unidades físicas independentes ou especiais e não são de livre acesso para atendimento individual ou coletivo. Devem contribuir para a integralidade do cuidado aos usuários do SUS ${ }^{4}$, auxiliando no aumento da capacidade de análise e de intervenção sobre problemas e necessidades de saúde (ANJOS et al., 2013).

Este serviço, em Reriutaba-CE é recente, ainda em processo de organização de suas práticas, que tiveram

4 São exemplos de ações do NASF- discussão de casos, atendimento conjunto ou não, interconsulta, construção conjunta de projetos terapêuticos, educação permanente, intervenções no território e na saúde de grupos populacionais e da coletividade, ações intersetoriais, ações de prevenção e promoção da saúde, discussão do processo de trabalho das equipes e outras (ANJOS et al., 2013). 
início nos primeiros meses do ano de 2016, mas que já proporcionou impactos positivos para a saúde do município, resultando em reconhecimento especial pelos membros de outras equipes e usuários do SUS.

Com essas discussões, cabe destacar que todas as características, práticas, relações, demandas, dificuldades e desafios não são fatores separados, todos estão entrelaçados pelas questões sociais, profissionais e culturais. Com base nessas pautas, buscam-se perceber as inúmeras formas de ver a saúde, ampliando e as possibilidades e potencialidades no serviço público, na saúde do homem, na atenção básica, nas percepções acerca de álcool e outras drogas, práticas interdisciplinares e em articulação com os demais serviços.

\section{CONSIDERAÇÕES FINAIS}

A inserção de ações que contemplem a saúde do homem nas Unidades Básicas de Saúde da Família se revela um grande desafio. Mesmo a PNAISH tendo sido lançada pelo Ministério da Saúde, sua materialização precisa superar conceitos e concepções moralistas, estereotipadas e mudar o foco para impulsionar uma reorganização no serviço e propiciar planejamento, programação e execução de ações voltadas para essa clientela.

$\mathrm{Na}$ presente pesquisa, que visou analisar como profissionais de uma Estratégia de Saúde da Família realizam o cuidado ao homem, no município de Reriutaba-CE, observou-se que ainda devem ser realizadas muitas ações de saúde do homem, para que estes venham fazer parte, efetivamente, da estratégia de saúde da família.

Percebeu-se também que o conhecimento da saúde deste público, por parte dos entrevistados, pautase principalmente na urologia, com valorização da próstata e ênfase no uso prejudicial de álcool e outras drogas. Parte dessa dificuldade se relaciona a uma limitada concepção de gênero por parte dos profissionais, ligada à característica biológica e papel cultural na sociedade e a um desconhecimento das políticas de saúde, dentre essas a PNAISH. Outro aspecto é a pequena presença dos homens na Unidade de Saúde e a inércia da equipe em propor ações que possam estimular essa participação.

Em meio às dificuldades, os profissionais também listam potencialidades para o trabalho com este público, uma vez que o serviço dispõe de boa infraestrutura física e de profissionais para execução das ações. Enfatizam também a possibilidade do trabalho intersetorial e interdisciplinar a partir de parcerias dentro a rede de saúde, como NASF e CAPS, e também com instituições da própria comunidade, como o AA e o terço dos homens da igreja católica.

Conclui-se que a equipe conhece as possibilidades para que os homens tenham um cuidado integral em Reriutaba-CE, no entanto, faltam iniciativas para que essas reflexões e questionamentos possam ser realizados e para que a equipe possa se qualificar e superar dificuldades como o preconceito, baixa interdisciplinaridade e pouca participação dos homens, enfim, atitudes para materializar o campo das possibilidades e potencialidades para que a saúde do homem seja realmente efetivada.

\section{REFERÊNCIAS}

ANJOS, K.F. et al. Perspectivas e desafios do núcleo de apoio à saúde da família quanto às práticas em saúde. Saúde em Debate, v. 37, n. 99, p. 672-680, 2013 Disponível em: < http://www.scielo.br/pdf/ sdeb/v37n99/a15v37n99.pdf>.

BRASIL. Conselho Nacional de Saúde (CNS).

Resolução n 466, de 12 de dezembro de 2012.

Disponível em: < http://conselho.saude.gov.br/ resolucoes/2012/Reso466.pdf >. Acesso em: $14 \mathrm{de}$ dez. 2015.

BRASIL. Ministério da Saúde. Política Nacional de Atenção Integral à Saúde do Homem. Princípios e Diretrizes, 2008. Disponível em: < http://dtr2001. saude.gov.br/sas/PORTARIAS/ Port2008/PT-09CONS.pdf >. Acesso em: 20 de nov. 2015. 
CAMARGO, B.V. et al. Representações sociais de saúde e cuidado: um estudo multicêntrico sobre vulnerabilidade masculina. Temas em psicologia, v. 19, n. 01, p. 179-192, 2011. Disponível em: <http://pepsic.bvsalud.org/scielo.php?pid=S1413389X2011000100014\&script=sci_arttext $>$ Acesso em: 06 jan. 2016.

CRP. Conselho Regional de Psicologia da $6^{\mathrm{a}}$ Região - São Paulo. Álcool e Outras Drogas. São Paulo: CRPSP, 2011. Disponível em: <http://www.crpsp.org. $\mathrm{br} /$ portal/comunicacao/livro-alcool-drogas/crpspalcool-e-outras-drogas.pdf >. Acesso em: 10 de nov. 2015.

IBGE. Instituto Brasileiro de Geografia e Estatística. Censo Populacional de 29 de novembro de 2010. Disponível em: <https://censo2010.ibge.gov.br/ resultados.html>. Acesso em: 20 de nov. 2017.

MACHIN, R. et al. Concepções de gênero, masculinidade e cuidados em saúde. Ciência \& Saúde Coletiva, v. 16, n. 11, p. 4503-4512, 2011. Disponível em: < www.scielosp.org/pdf/csc/v16n11/ a23v16n11.pdf>. Acesso em: 20 de nov. 2015.

MAHALIK, J.R., BURNS, S.M., SYZDEK, M. Masculinity and perceived normative health behaviors as predictors of men's health behaviors. Social Science \& Medicine, v. 64, n.11, p. 2201-2209, 2007. Disponível em: <http://www.sciencedirect.com/ science/article/pii/S0277953607000846>. Acesso em: 22 de nov. 2017.
MINAYO, M.C.S. Os desafios do conhecimento: pesquisa qualitativa em saúde. Ed. 9a , São Paulo: Hucitec, 2006.

SCHRAIBER, L.B. Necessidades de saúde, políticas públicas e gênero: a perspectiva das práticas profissionais. Ciência \& Saúde Coletiva, v. 17, n. 10, p. 2635-2644, 2012. Disponível em: <www.scielo. br/pdf/csc/v17n10/13.pdf >. Acesso em: 10 de nov. 2015.

SCHRAIBER, L.B. et al. Necessidades de saúde e masculinidades: atenção primária aos cuidados dos homens. Cad. Saúde Pública, v. 26, n. 05, p. 961-970, 2010. Disponível em: <http://www.scielosp.org/pdf/ csp/v26n5/18.pdf>. Acesso em: 13 jan. 2016

SCLIAR, M. História do Conceito de Saúde. Physis: Rev. Saúde Coletiva, v. 17, n. 1, p. 29-41, 2007. Disponível em: < http://www.scielo.br/pdf/physis/ v17n1/v17n1a03.pdf >. Acesso em: 14 abr. 2016.

SOUZA, I.C.W.; RONZANI, T.M. Álcool e drogas na atenção primária: avaliando estratégias de capacitação. Psicologia em estudo, Maringá, v. 17, n. 2, p. 237-246, 2012. Disponível em: <http://www.scielo.br/scielo.php?pid=S141373722012000200007 \&script=sci_abstract\&tlng=pt $>$. Acesso em: 10 de mar. 2015. 


\begin{abstract}
1 Psicóloga pela Universidade Federal do Piauí - UFPI; Mestranda em Políticas Públicas - UFPI; Especialista em Saúde Coletiva (residência multiprofissional) pela Escola de Saúde Pública do Ceará - ESP. E-mail: mayaracapereira@gmail.com.
\end{abstract}

2 Fisioterapeuta pelo Centro Universitário Leão Sampaio - UNILEÃO; Especialista em Saúde Coletiva (residência multiprofissional) pela Escola de Saúde Pública do Ceará - ESP. E-mail: marbiaaraujo@gmail.com.

3 Assistente Social pela Universidade Estadual do Ceará - UECE; Doutora em Educação pela Universidade Federal do Ceará - UFC; Professora do Instituto Federal de Educação, Ciência e Tecnologia do Ceará - IFCE, campus Iguatu. E-mail: adriana.as.ce@gmail.com. 
